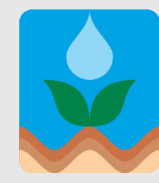

Revista Brasileira de Engenharia Agrícola e Ambiental

Campina Grande, PB, UAEA/UFCG - http://www.agriambi.com.br Protocolo 296.13 - 18/09/2013 • Aprovado em 27/06/2014

\title{
Diseño y predicción del funcionamiento de inyectores Venturi en riego localizado
}

\author{
Juan Manzano ${ }^{1}$, Benito M. de Azevedo², Guilherme V. do Bomfim³, \\ Álvaro Royuela ${ }^{4}$, Carmen V. Palau ${ }^{5}$ \& Thales V. de A. Viana ${ }^{6}$ \\ ${ }^{1}$ DIRA/UPV, Valencia, España. E-mail: juamanju@agf.upv.es \\ ${ }^{2}$ CCA/DENA/UFC, Fortaleza, CE. E-mail: benitoazevedo@hotmail.com \\ ${ }^{3}$ CCA/DENA/UFC, Fortaleza, CE. E-mail: guile01@ig.com.br (Autor correspondente) \\ ${ }^{4}$ DIRA/UPV, Valencia, España. E-mail: aroyuela@agf.upv.es \\ ${ }^{5}$ DIRA/UPV, Valencia, España. E-mail: virpaes@agf.upv.es \\ ${ }^{6} \mathrm{CCA} / \mathrm{DENA} / \mathrm{UFC}$, Fortaleza, CE. E-mail: thales@ufc.br
}

\section{Palabras-clave:}

dinámica de fluidos computacional

RANS

quimigación

\section{R E S U M E N}

En la Universitat Politècnica de València, Valencia, España, se ha estudiado el diseño y funcionamiento de cuatro prototipos del inyector Venturi con técnicas de Dinámica de Fluidos Computacional (CFD), comparándo las con ensayos en laboratorio. Para cada prototipo, las geometrías definidas y analizadas han permitido simular el funcionamiento sin (G1) y con inyección (G2) para quimigación. En el caso G1, se presentan los gráficos del perfil de presiones en el eje del inyector para diversas velocidades, así como la distribución del campo de presiones y de la evolución de las diferencias de presión y pérdidas de carga frente al caudal principal. Para comparar los resultados obtenidos con CFD frente al resultado experimental, se calculó el error relativo. En el caso G2, se obtuvo la representación gráfica del el caudal de inyección frente al caudal principal. Las técnicas CFD exigen un buen ajuste del modelo para dar un resultado aceptable. Son interesantes para comparar geometrías, analizar sus variantes, realizar prediseños y aproximar ordenes de magnitud, pero es recomendable su ensayo en laboratorio para validar los resultados.

\section{Key words:}

computational fluid dynamics

RANS

chemigation

\section{Design and prediction performance of Venturi injectors in drip irrigation}

\begin{abstract}
A B S T R A C T
The design and prediction performance of four Venturi injector prototypes have been studied using Computational Fluid Dynamics (CFD) techniques. Results were compared with experimental tests carried out in the laboratory of the Universitat Politècnica de València, Valencia, Spain. The analysed and selected geometries for each prototype were used to simulate the operation without nutrient injection (G1) and with nutrient injection (G2). In first case (G1), the results were presented in the form of pressure profile at the injector axe under different velocities and the pressure distribution in the whole geometry. Additionally, this paper analysed the evolution of pressures and head loss versus main water flow in the different prototypes. The relative error was estimated to compare CFD and experimental results. The second case (G2), the graphical representation for the relations between the nutrient aspiration flow and water main flow were obtained for numerical and experiment approaches. In conclusion, CFD techniques appear as a suitable tool for the analysis of the Venturi injector operation, but its validation with experimental data is recommended.
\end{abstract}

\section{INTRODUCCIÓN}

Las técnicas computacionales de fluidos o la Dinámica de Fluidos Computacional (traducción más próxima al término inglés Computational Fluid Dynamics, CFD), se ocupan de obtener soluciones numéricas de problemas de mecánica de fluidos con el apoyo de ordenadores (Wendt, 2009). Se basan en la resolución numérica de las ecuaciones fundamentales de conservación de materia, energía y cantidad de movimiento en un dominio concreto discretizado (geometría); es decir, convertido en una malla de puntos (volúmenes o elementos finitos). Como resultado, se obtienen los valores de todas las variables características del sistema (presión, velocidad, temperatura, composición, etc.) en cada punto de la malla de cálculo y en función del tiempo (Manzano, 2008).

En Ingeniería Hidráulica, son muy numerosos los ejemplos de aplicación de técnicas CFD para la modelación y mejora de sistemas (Davis \& Stewart, 2002). En los sistemas de riego existen ejemplos en emisores de riego localizado y sistemas de inyección de fertilizantes (Wei et al., 2006; Palau-Salvador et 
al., 2008; Yeoh et al., 2012). Otros trabajos con estas técnicas incluyen la análisis del aumento de diámetro en tuberías (Guo et al., 2002), evaluación de bombas centrifugas (Cipolla et al., 2011), evaluación de sensores en invernaderos (Norton et al., 2007; Teruel, 2010).

Otro campo donde existe una amplia experiencia aplicada es en el estudio de la cavitación (Hatano et al., 2014). Esta es una línea de investigación que en la actualidad es muy activa, presentándose numerosos trabajos y aportaciones sobre modelos de cavitación. Es muy habitual el uso de toberas o de tubos Venturi para realizar los ensayos experimentales y para validar los modelos de cavitación (Lindau et al., 2002; Singhal et al., 2002). También se ha analizado el comportamiento no estacionario de la formación de vapor con un Venturi rectangular (Delgosha et al., 2003). Los modelos de turbulencia empleados en prácticamente la totalidad de estos trabajos son RANS [ $\mathrm{k}-\varepsilon, \mathrm{k}-\omega \mathrm{y}$ RSM], aunque también existen algunos ejemplos válidos con modelos DNS (Xing \& Frankel, 2002).

El software utilizado en las técnicas CFD es amplio, existiendo paquetes comerciales, generales o específicos, que integran todo el proceso de diseño, desde la construcción de la geometría a analizar y su descomposición en una malla (preprocesado), el procesado o la resolución del problema (modelo físico, método numérico y algoritmo de solución) hasta el análisis y representación de los resultados (postprocesado). Algunos de los códigos comerciales usados comúnmente en aplicaciones de Ingeniería Hidráulica son: Preprocesado (Pro-Engineer, Gridpro, ICEM-CFD, Gambit, etc.); Procesado (Flow-3D, Star-CD, CFD-ACE+, Fluent, TascFlow-CFX, etc.); Postprocesado (Tecplot, Fieldwiew, Ensight, etc.).

Las técnicas CFD constituyen una herramienta muy potente en el diseño e investigación hidráulica, pero deben ser aplicadas adecuadamente. El empleo de un modelo robusto y con un programa informático contrastado, no suponen por sí solo una garantía de precisión, sino que requiere la validación de los resultados por medio de experimentación clásica. En el presente trabajo se comprueba si las técnicas de Dinámica de Fluidos Computacional (Computational Fluid Dynamics, CFD) son adecuadas para el diseño de nuevos inyectores Venturi y la predicción del funcionamiento de modelos comerciales.

\section{MATERIAL Y Métodos}

El banco de ensayos está situado en el laboratorio de Hidráulica y Riego Localizado del Departamento de Ingeniería Rural y Agroalimentaria de la Universitat Politècnica de València, Valencia, España (39 $29^{\prime} \mathrm{N}, 0^{\circ} 23^{\prime} \mathrm{W}$ y $20 \mathrm{~m}$ ).
Se han experimentado, en 2008, cuatro prototipos de inyectores Venturi instalados en serie con las siguientes dimensiones (Tabla 1).

El ensayo de cada unidad (Figura 1) se ha desarrollado en dos fases. En la primera (E1), con la aspiración cerrada, se mantuvo constante la presión en la entrada del inyector $\left(\mathrm{P}_{1}\right)$ y se incrementó progresivamente el caudal $\left(Q_{1}\right)$ hasta que se detectó la cavitación en la garganta. En la segunda fase (E2), la toma de datos se realizó de forma semejante a la fase anterior. La diferencia con la fase E1 reside en que la adquisición de datos comenzó cuando se alcanzaron presiones negativas, registrándose valores con la válvula de aspiración cerrada y abierta en cada regulación.

Para la comparación de funcionamiento de los prototipos con la técnica $\mathrm{CFD}$, se obtuvieron las siguientes variables: presión en la entrada $\left(\mathrm{P}_{1}\right)$, en la garganta $\left(\mathrm{P}_{2}\right)$ y en la salida $\left(\mathrm{P}_{3}\right)$; presión diferencial (DP) entre la entrada y la garganta $\left(\mathrm{P}_{1}-\mathrm{P}_{2}\right)$; caudal principal $\left(\mathrm{Q}_{1}\right)$ : caudal de entrada en la tobera del inyector; caudal de inyección (q): caudal inyectado en la garganta por la tubería de aspiración; pérdidas totales en el inyector $\left(\Delta \mathrm{h}_{\mathrm{v}}\right)$ : diferencia de presión entre la garganta y la salida $\left(\mathrm{P}_{1}-\mathrm{P}_{3}\right)$; velocidad $\left(\mathrm{V}_{1}\right)$. Las unidades utilizadas han sido: para $\mathrm{P}_{1}, \mathrm{P}_{2}, \mathrm{P}_{3}$, $\mathrm{DP}, \Delta \mathrm{h}_{\mathrm{v}}$ y $\mathrm{P}_{3}-\mathrm{P}_{2}, \mathrm{kPa}$.; para $\mathrm{Q}_{1}, \mathrm{~L} \mathrm{~s}^{-1}$; para $\mathrm{q}, \mathrm{L} \mathrm{h}^{-1}$; para $\mathrm{V}_{1}, \mathrm{~m} \mathrm{~s}^{-1}$.

$\mathrm{La}$ adquisición de datos estaba totalmente automatizada mediante el empleo de transductores de presión (puntos 3, 5 y 7 de la Figura 1), un caudalímetro electromagnético para $Q_{1}$ (punto 1, Figura 1) y una célula de carga para la medida del volumen de solución inyectada dividido por el tiempo de cada periodo de medida, q (punto 9, Figura 1). Las señales analógicas

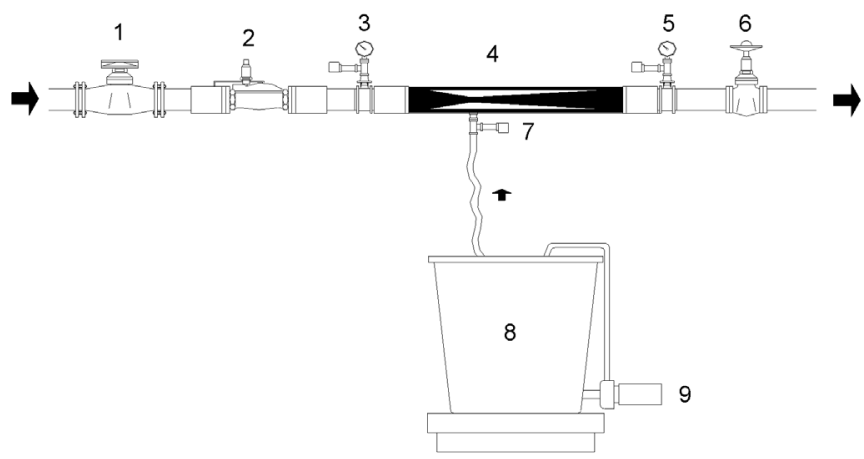

Figura 1. Esquema del ensayo experimental. 1 caudalímetro, 2 - válvula reductora de presión, 3 sensor de presión (P1), 4 - inyector Venturi, 5 - sensor de presión (P3), 6 - válvula de regulación, 7 - válvula de aspiración y sensor de presión (P3), 8 - solución de inyección, 9 - sistema de nivel constante y pesada solución de inyección

Tabla 1. Dimensiones de los prototipos de inyectores Venturis

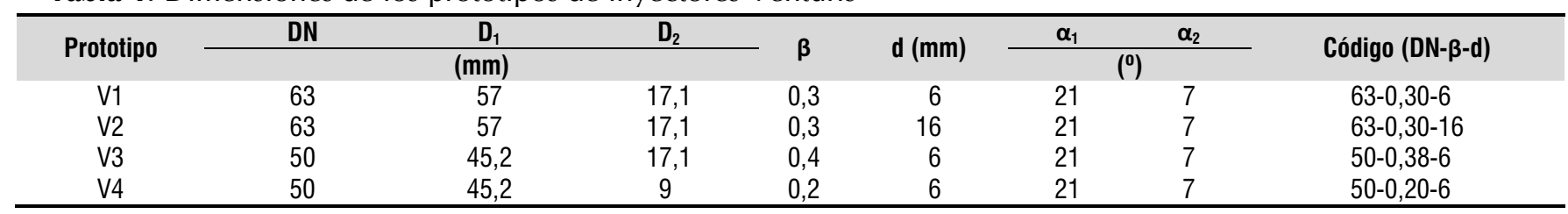

DN - diámetro nominal de la tubería en que se inserta el inyector; $D_{1}$ - diámetro de la tobera; $D_{2}$ - diámetro de la garganta; $\beta$ - relación de diámetros $\left(D_{2} / D_{1}\right) ; d$ - diámetro de la tubería de aspiración $\alpha_{1}$ - ángulo de la tobera; $\alpha_{2}$ - ángulo del difusor 
procedentes de los diferentes sensores eran adquiridas mediante una tarjeta de National Instriuments PCI 6023E. Finalmente el procesado conjunto de los datos para cada punto de medición se realizó por medio de una aplicación informática desarrollada en LabVIEW 7.1. Cada medición estaba compuesta por el registro simultáneo de cada variable (presión o caudal) en su respectivo punto. Cada medida tenía $10 \mathrm{~s}$ de duración. En cada segundo, fueron registrados el promedio de 100 muestras, es decir, 10 promedios por medida. El valor definitivo de cada variable medida fue la media final de estos 10 promedios.

Para el desarrollo de este trabajo con CFD se han seleccionado tres programas. Para la construcción de la geometría y el mallado, el programa empleado ha sido el GAMBIT 2.2.30. El solver FLUENT 6.2.16 fue el programa de procesado. Finalmente, para el postprocesado, ha sido utilizado el programa TECPLOT 360.

Para cada prototipo, las geometrías definidas y analizadas con el GAMBIT han sido dos: una G1, para simular el funcionamiento sin inyección (Figura 2A); otra G2, teniendo en cuenta la inyección (Figura 2B). Reproducir con detalle la geometría de la tubería de aspiración (Figura 2C) es muy importante puesto que tendrá una gran influencia en el caudal inyectado.

Otras dos posibles geometrías analizadas se presentan en la Figura 3.

La geometría de la Figura 3A se desechó por requerir tiempos de cálculo muy grandes, sin mejora en la calidad de los resultados. La geometría de la Figura 3B presentó un ahorro de tiempo de procesado respecto a la Figura 2A, pero con resultados algo más distantes a los experimentales.

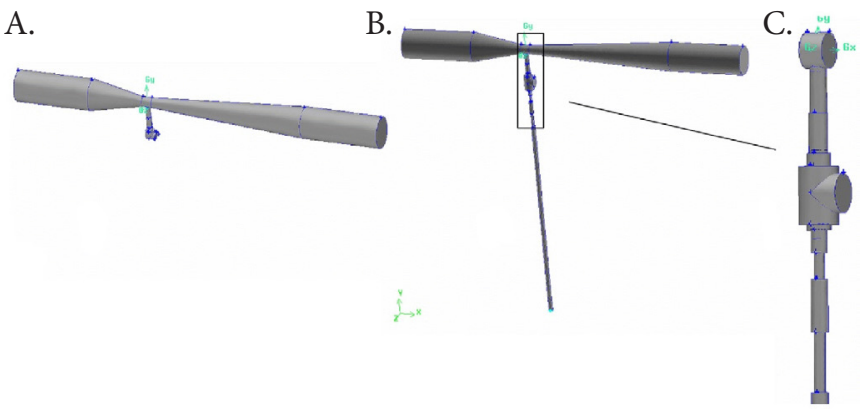

Figura 2. Geometría G1 (sin inyección (A)), G2 (con inyección (B)) y detalle del tubo de aspiración (C)

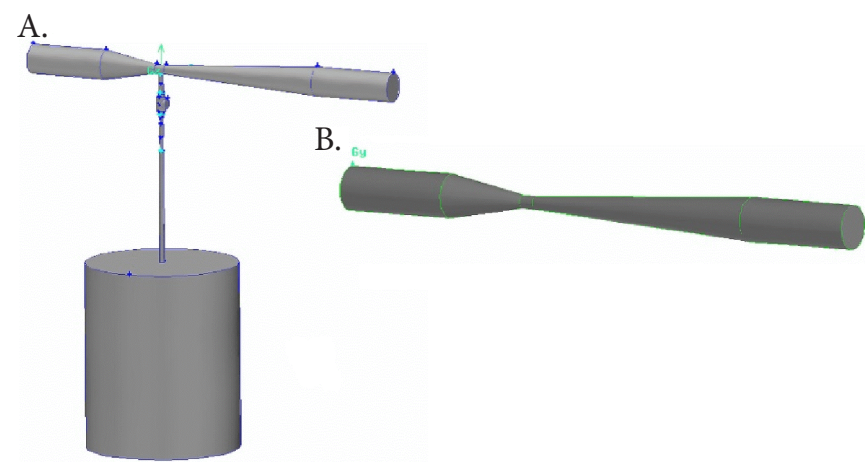

Figura 3. Geometría G2, con depósito de aspiración (A) y G1, sin aspiración (B)
Tras la definición de la geometría y de la malla, se asignaron a las superficies exteriores de cada geometría las condiciones de contorno que fueron utilizadas durante el análisis (pared, velocidad de entrada, presión de salida y presión de entrada).

Para la obtención de la relación de presiones, se simuló la geometría G1 de cada prototipo incrementando la velocidad $\mathrm{V}_{1}$, a partir de un valor mínimo de $0,5 \mathrm{~m} \mathrm{~s}^{-1}$. En todos los casos, se estableció una presión en la sección de salida, $\mathrm{P}_{3}$, constante. La velocidad de entrada al inyector fue incrementada progresivamente hasta la aparición de presiones de vapor en el eje del inyector.

Para la obtención de estas relaciones, no se consideró inicialmente la cavitación. Para tenerla presente, ajustando así el resultado al fenómeno real, se volvió a analizar la geometría a partir de aquella velocidad en que aparecerán presiones de vapor en cualquier punto. Este segundo ensayo se realizó en dos etapas: primero, se fijaron las presiones a la entrada y salida, $\mathrm{P}_{1}$ y $\mathrm{P}_{3}$, sin considerar la cavitación, $\mathrm{y}$ se registró el caudal circulante; a continuación, se activó el modelo de cavitación, manteniendo constante estas presiones y registrando el nuevo caudal.

Cada análisis con FLUENT se ha llevado a cabo con dos modelos de turbulencia K- $\varepsilon$ (RNG) y RSM y dos niveles de discretización de primer orden, $\mathrm{k}-\varepsilon 1$ y RSM 1 , y segundo orden, k- $\varepsilon 2$ y RSM 2. Para cada prototipo y modelo de turbulencia, se representó (p.e. modelo RSM 1 en las Figuras), el gráfico del perfil de presiones en el eje del inyector para ciertas velocidades y una distribución del campo de presiones, para la velocidad máxima, antes y después de incluir la cavitación. También se obtuvo el gráfico de la evolución de las diferencias de presión, DP, y pérdidas de carga, $\Delta \mathrm{h}_{\mathrm{v}}$ y $\mathrm{P}_{3}-\mathrm{P}_{2}$, frente al caudal principal, $\mathrm{Q}_{1}$, para cada punto de funcionamiento modelizado.

Para poder comparar los resultados obtenidos con los modelos CFD frente al resultado experimental, se calculó el error relativo para cada modelo en el rango de caudales principales, que viene definido por las velocidades, $\mathrm{V}_{1}$, máxima y mínima consideradas. El resultado de los valores obtenidos fue tabulado, expresándose los errores en porcentaje (Error modelo = valor experimental/valor del modelo x 100).

Para la geometría G2 (aspiración inferior), se obtuvieron los caudales inyectados, $\mathrm{q}$, y su relación con el caudal principal, $Q_{1}$. El registro de datos se iniciaba a partir de aquella velocidad con la que comenzaban a producirse presiones negativas en la garganta; posteriormente, se incrementaba progresivamente la velocidad hasta la aparición de la cavitación. Durante todo el ensayo, se mantuvo fija la presión en la salida de Venturi, $\mathrm{P}_{3}$.

Al igual que se hizo en la fase E1, el procesado incluyó el efecto de la cavitación, realizándose en dos etapas e introduciendo como condiciones de contorno las presiones en la entrada y la salida, $\mathrm{P}_{1}$ y $\mathrm{P}_{3}$; obteniéndose la representación gráfica de los caudales de entrada y de aspiración, $Q_{1}$ y q, en cada prototipo.

En las siguientes Tablas 2 y 3 se presenta el resumen de los prototipos modelados. 
Tabla 2. Resumen modelado de los prototipos en la fase G1

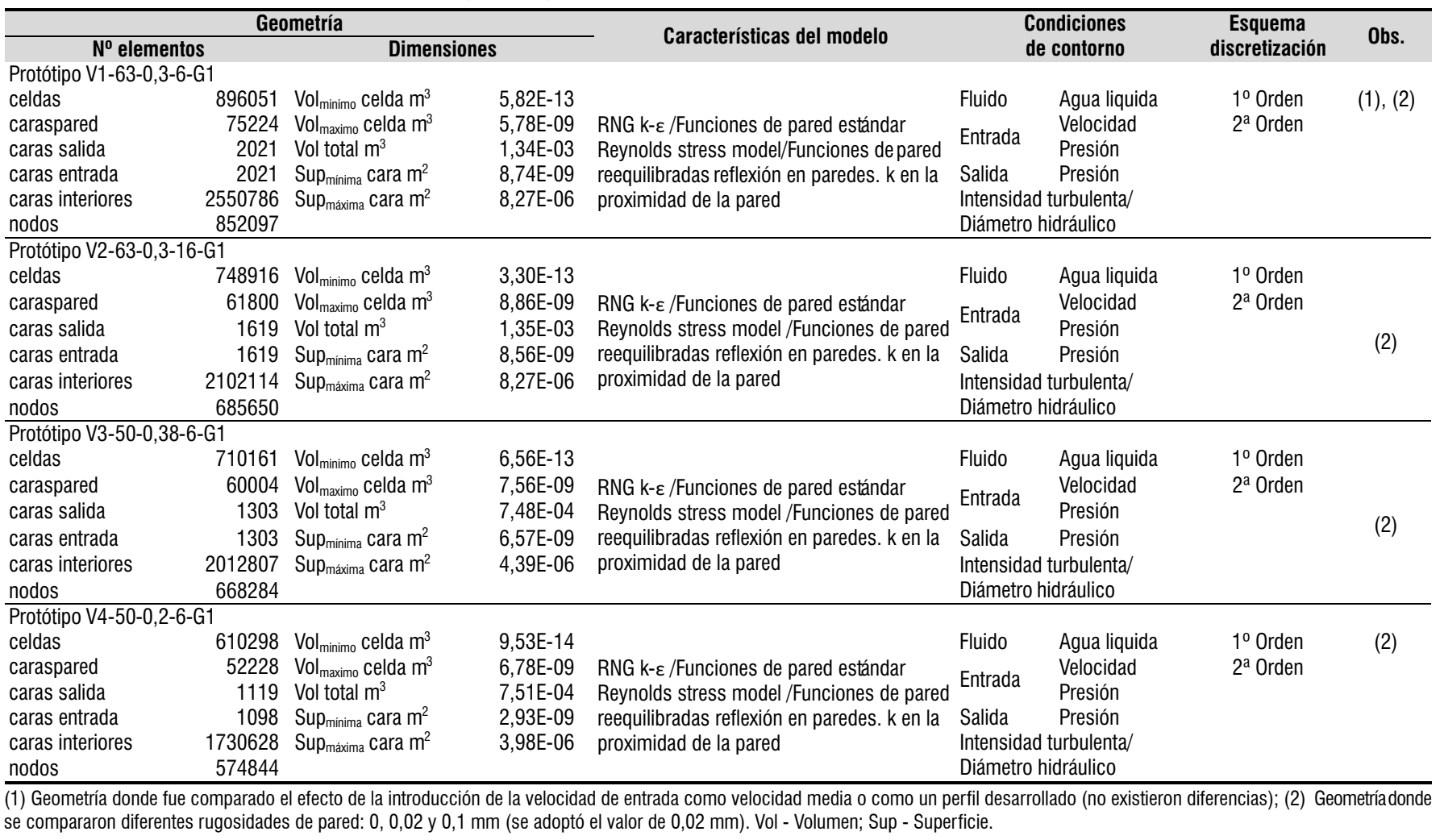

Tabla 3. Resumen modelado de los prototipos en la fase G2

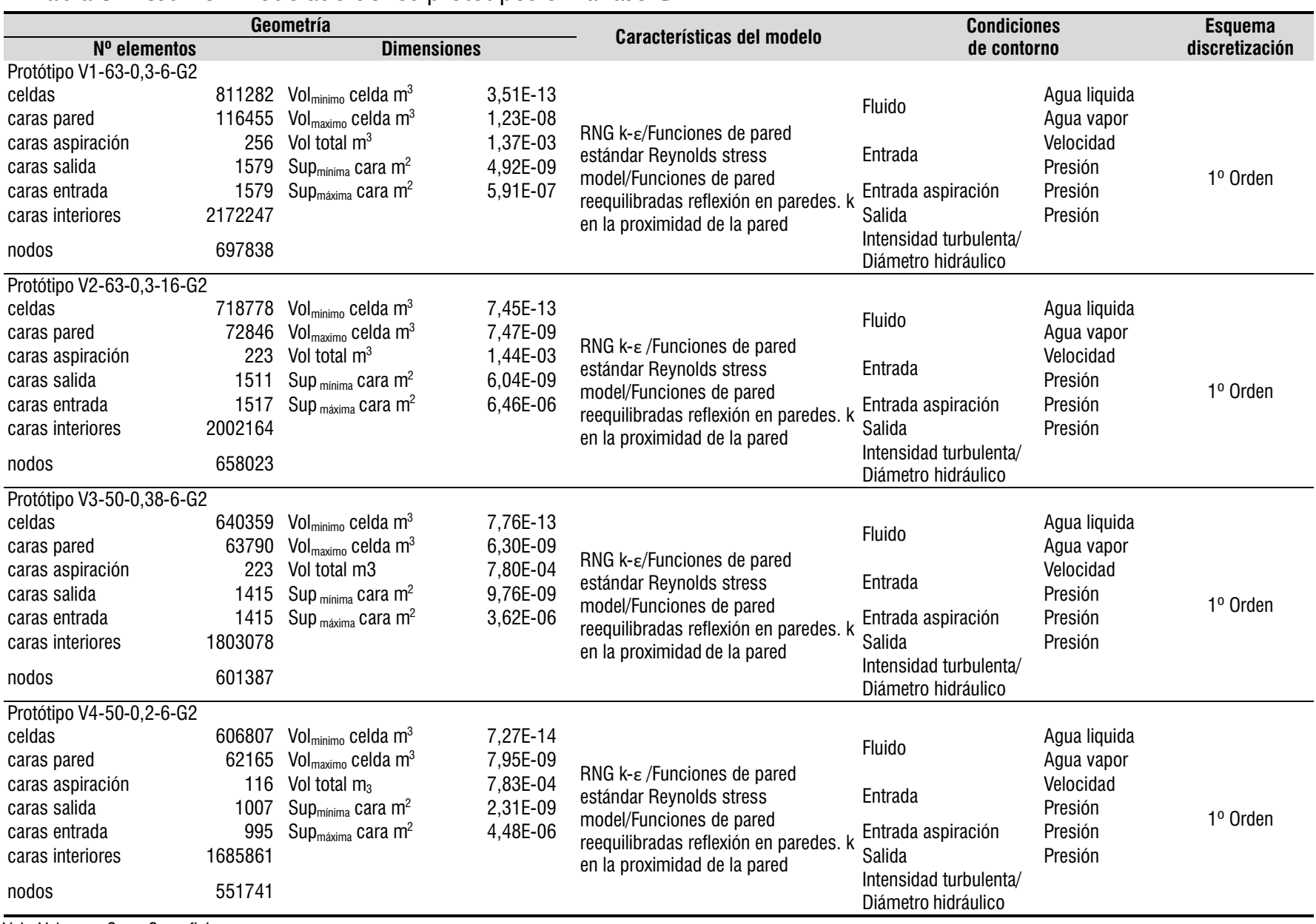

Vol - Volumen; Sup - Superficie. 


\section{Resultados y Discusión}

\section{Fase E1 - sin inyección}

Los resultados del perfil de presiones y la distribución del campo de presiones obtenidos para los prototipos V1-63-0,3-6, V2-63-0,3-16, V3-50-0,38-6 y V4-50-0,2-6 se muestran en las respectivas Figuras 4, 5, 6 y 7 .

En las respectivas Figuras 8, 9, 10 y 11 se muestran las diferencias de presión y pérdidas de carga frente al caudal principal y la comparativa de los modelos CFD con el ensayo, en los prototipos V1-63-0,3-6, V2-63-0,3-16, V3-50-0,38-6 y V4-50-0,2-6.

En las Tablas 4, 5, 6 y 7 se muestran los resultados del error relativo entre los modelos CFD para los prototipos
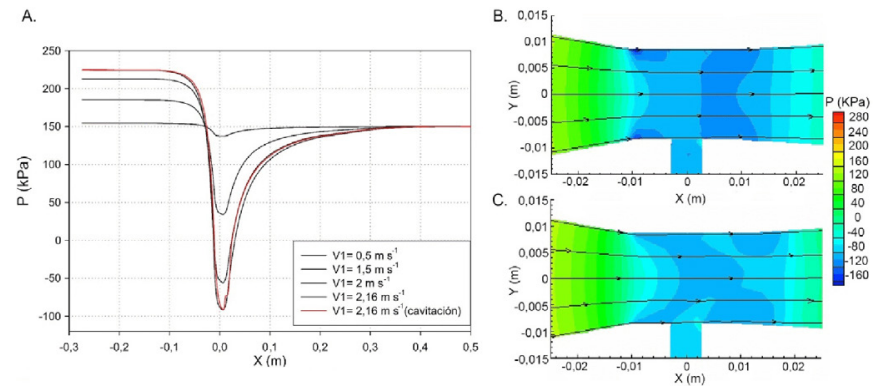

Figura 4. Perfil de presiones en el eje para distintas velocidades de entrada (A) y distribución de presiones sin cavitación (B) y con cavitación $(\mathrm{C})$, según el modelo RSM 1, para el prototipo V1-63-0,3-6
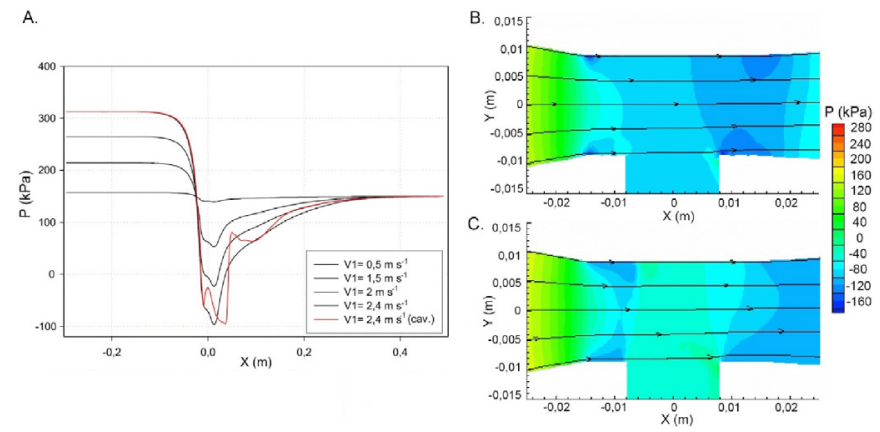

Figura 5. Perfil de presiones en el eje para distintas velocidades de entrada (A) y distribución de presiones sin cavitación (B) y con cavitación (C), según el modelo RSM 1, para el prototipo V2-63-0,3-16
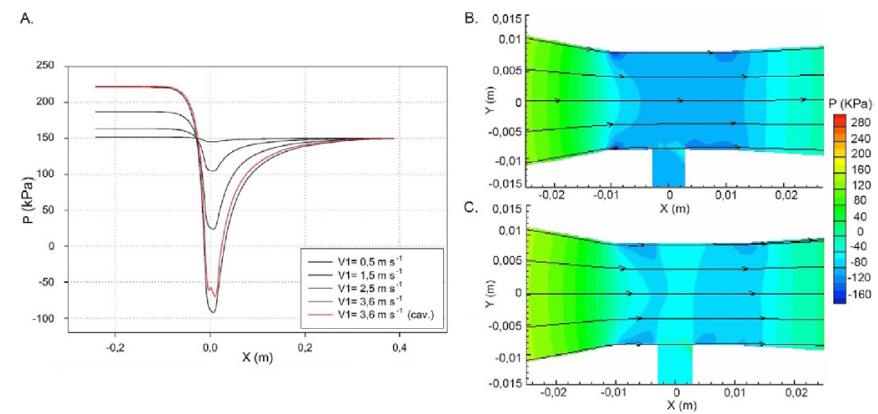

Figura 6. Perfil de presiones en el eje para distintas velocidades de entrada (A) y distribución de presiones sin cavitación (B) y con cavitación $(\mathrm{C})$, según el modelo RSM 1, para el prototipo V3-50-0,38-6
V1-63-0,3-6, V2-63-0,3-16, V3-50-0,38-6 y V4-50-0,2-6, respectivamente.

Conforme las Tablas 4-7, en algunos casos el error entre simulación y ensayo es alto, concretamente para velocidades bajas o altas (próximas a cavitación) pero en la zona central del rango de trabajo los errores serian aceptables. A partir de las Figuras y de las tablas de error, puede afirmarse que el modelo $\mathrm{k}-\varepsilon$ predice una distribución de velocidad menos simétricas que el RSM.

La existencia de una toma-orificio en la garganta, aún sin inyección, da lugar a una alteración del flujo tanto mayor cuanto
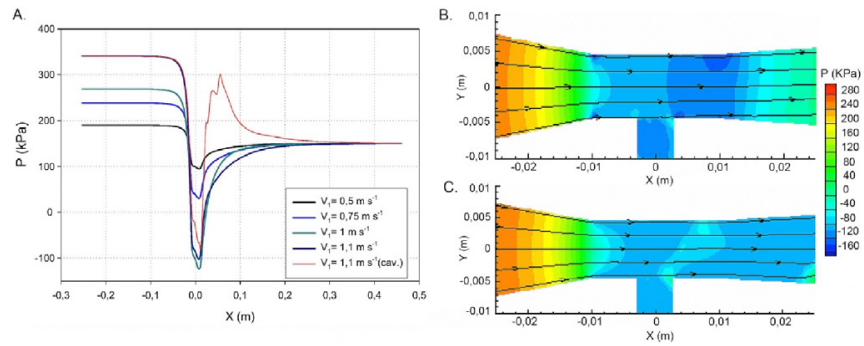

Figura 7. Perfil de presiones en el eje para distintas velocidades de entrada (A) y distribución de presiones sin cavitación (B) y con cavitación $(C)$, según el modelo RSM 1, para el prototipo V4-50-0,2-6
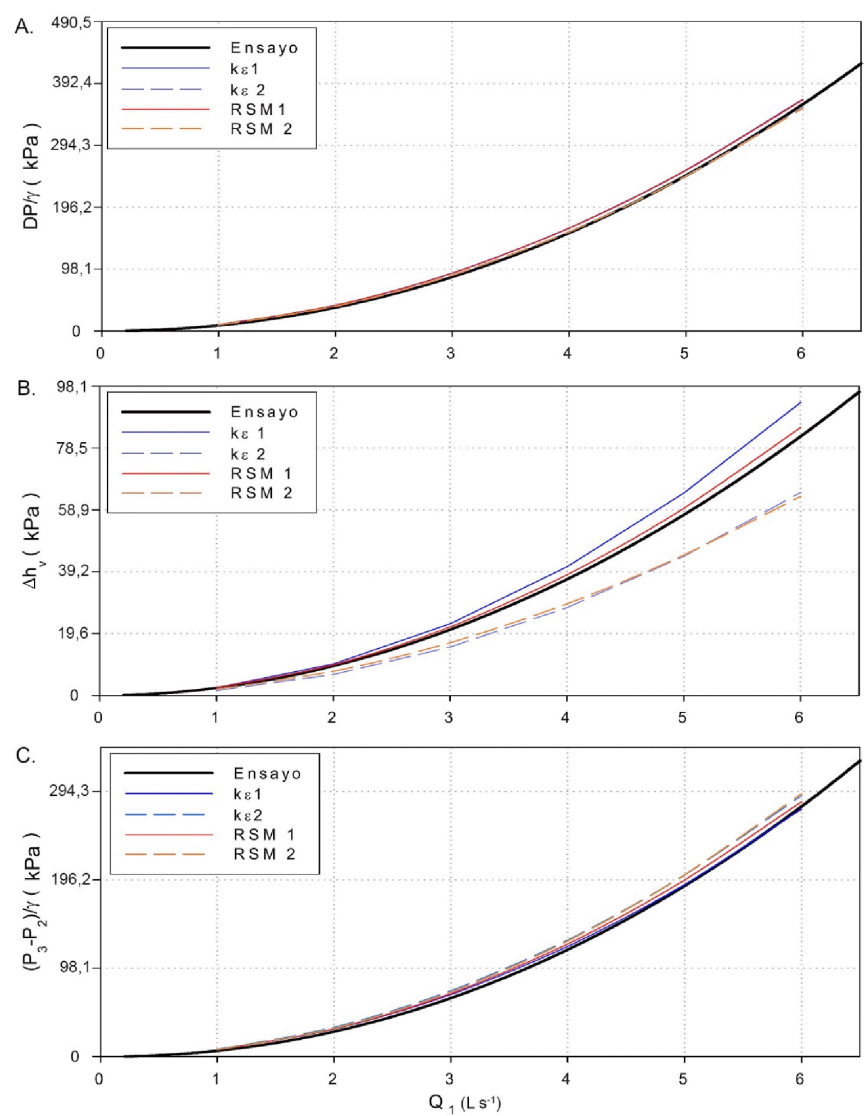

Figura 8. Presión diferencial entre la entrada y la garganta (DP) (A), pérdidas totales en el inyector $\left(\Delta \mathrm{h}_{\mathrm{v}}\right)$ (B) y presión diferencial entre la garganta y la salida $\left(\mathrm{P}_{3}-\mathrm{P}_{2}\right)(\mathrm{C})$ frente al caudal principal $\left(\mathrm{Q}_{1}\right)$ y comparativa de los modelos CFD con el ensayo, para el prototipo V1-63-0,3-6 

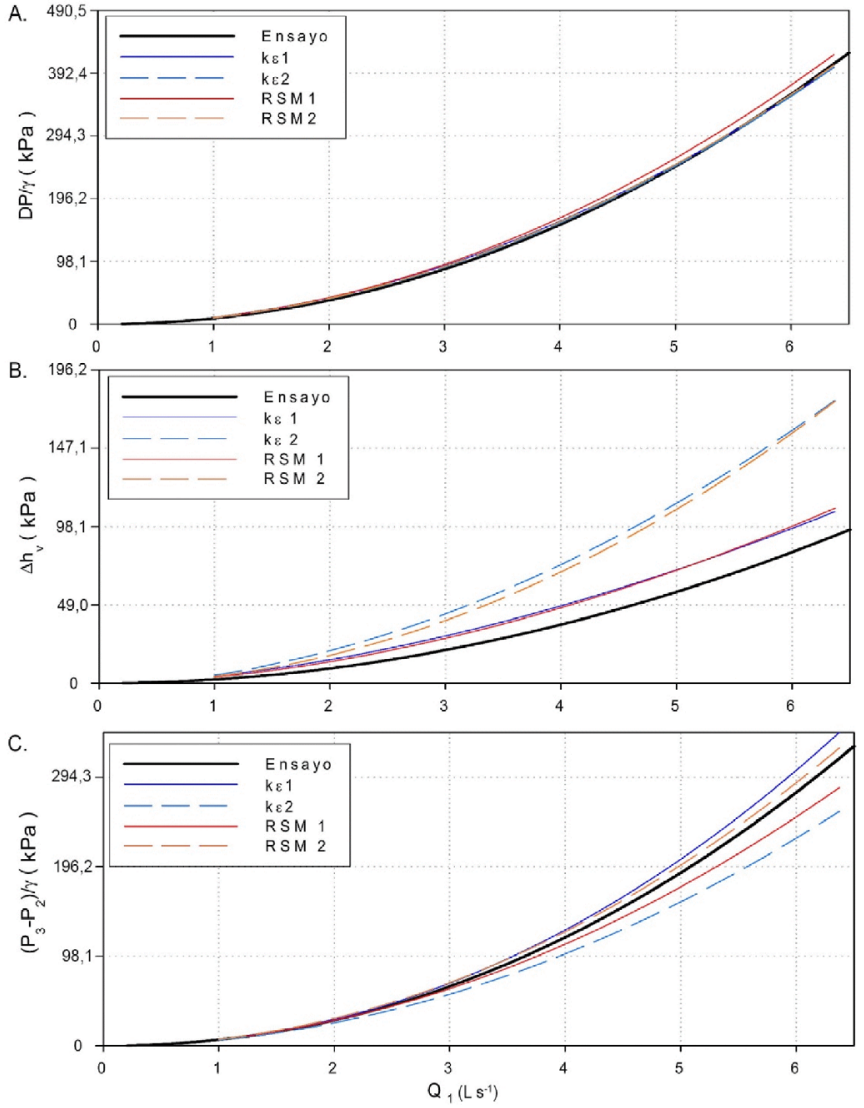

Figura 9. Presión diferencial entre la entrada y la garganta (DP) (A), pérdidas totales en el inyector $\left(\Delta \mathrm{h}_{\mathrm{v}}\right)$ (B) y presión diferencial entre la garganta y la salida $\left(\mathrm{P}_{3}-\mathrm{P}_{2}\right)(\mathrm{C})$ frente al caudal principal $\left(\mathrm{Q}_{1}\right)$ y comparativa de los modelos CFD con el ensayo, para el prototipo V2-63-0,3-16
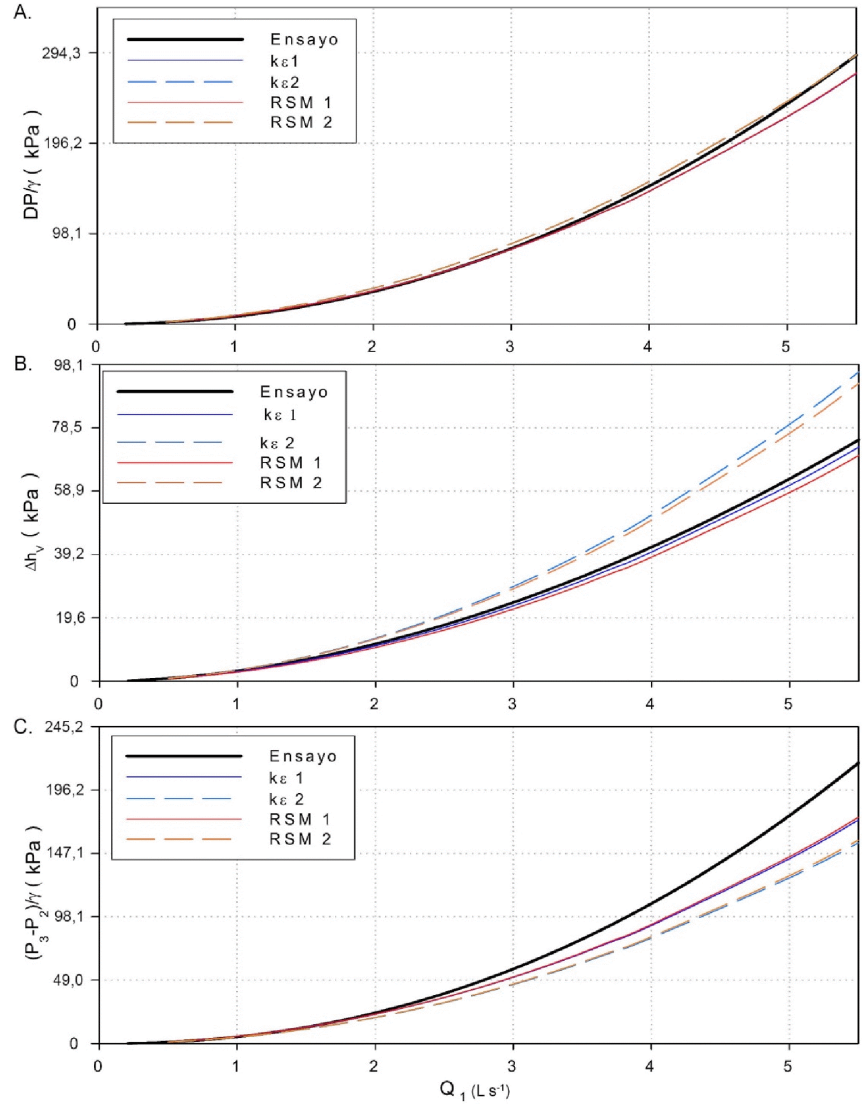

Figura 10. Presión diferencial entre la entrada y la garganta (DP) (A), pérdidas totales en el inyector $\left(\Delta h_{v}\right)$ (B) y presión diferencial entre la garganta y la salida $\left(\mathrm{P}_{3}-\mathrm{P}_{2}\right)(\mathrm{C})$ frente al caudal principal $\left(\mathrm{Q}_{1}\right)$ y comparativa de los modelos CFD con el ensayo, para el prototipo v3-50-0,38-6

Tabla 4. Error relativo de los modelos CFD, en el prototipo V1-63-0,3-6

\begin{tabular}{|c|c|c|c|c|c|c|c|c|c|c|c|c|c|}
\hline \multirow{2}{*}{$\begin{array}{c}V_{1} \\
\left(\mathrm{~m} \mathrm{~s}^{-1}\right)\end{array}$} & \multirow{2}{*}{$\begin{array}{c}\mathbf{a}_{1} \\
\left(L s^{-1}\right)\end{array}$} & \multicolumn{4}{|c|}{ DP (\%) } & \multicolumn{4}{|c|}{$\Delta h_{v}(\%)$} & \multicolumn{4}{|c|}{$P_{3}-P_{2}(\%)$} \\
\hline & & $k-\varepsilon 1$ & $k-\varepsilon 2$ & RSM 1 & RSM 2 & $k-\varepsilon 1$ & $k-\varepsilon 2$ & RSM 1 & RSM 2 & $k-\varepsilon 1$ & $k-\varepsilon 2$ & RSM 1 & RSM 2 \\
\hline 0,5 & 1,27 & $13,1^{*}$ & $9,0^{*}$ & $13,0^{*}$ & $9,2^{*}$ & $4,3^{\star \star}$ & $31,8^{*}$ & $5,0^{*}$ & $15,4^{* *}$ & $15,4^{*}$ & $23,2^{*}$ & $15,5^{*}$ & $17,2^{*}$ \\
\hline 1,9 & 4,75 & - & - & - & $0,1^{* *}$ & - & - & - & - & - & - & - & - \\
\hline 2,0 & 5,00 & - & $0,1^{* *}$ & - & - & - & - & - & - & - & - & - & - \\
\hline 2,2 & 5,50 & - & - & _ & - & - & - & - & - & $0,2^{* *}$ & & - & \\
\hline 2,5 & 6,37 & $2,0^{* *}$ & - & $1,8^{* *}$ & - & $13,5^{\star}$ & $21,2^{* *}$ & $3,4^{* *}$ & $23,4^{*}$ & - & $3,7^{\star *}$ & $1,4^{* *}$ & $4,7^{\star *}$ \\
\hline
\end{tabular}

$V_{1}$ - velocidad; $Q_{1}$ - caudal principal; DP - presión diferencial entre la entrada y la garganta $\left(P_{1}-P_{2}\right) ; \Delta h_{v}$ - pérdidas totales en el inyector $\left(P_{1}-P_{3}\right) ;\left(P_{3}-P_{2}\right)$ - presión diferencial entre la garganta y la salida; "error máximo; **error mínimo

Tabla 5. Error relativo de los modelos CFD, en el prototipo V2-63-0,3-16

\begin{tabular}{|c|c|c|c|c|c|c|c|c|c|c|c|c|c|}
\hline \multirow{2}{*}{$\begin{array}{c}\mathbf{V}_{1} \\
\left(\mathrm{~m} \mathrm{~s}^{-1}\right)\end{array}$} & \multirow{2}{*}{$\begin{array}{c}\mathbf{a}_{1} \\
\left(\mathrm{Ls}^{-1}\right)\end{array}$} & \multicolumn{4}{|c|}{ DP (\%) } & \multicolumn{4}{|c|}{$\Delta h_{v}(\%)$} & \multicolumn{4}{|c|}{$P_{3}-P_{2}(\%)$} \\
\hline & & $k-\varepsilon 1$ & $k-\varepsilon 2$ & RSM 1 & RSM 2 & $k-\varepsilon 1$ & $k-\varepsilon 2$ & RSM 1 & RSM 2 & $k-\varepsilon 1$ & $k-\varepsilon 2$ & RSM 1 & RSM 2 \\
\hline 0,5 & 1,27 & $3,3^{* *}$ & $8,6^{* \star}$ & $4,48^{* *}$ & $6,8^{* *}$ & $2,4^{\star}$ & $24,5^{* *}$ & $10,2^{*}$ & $6,2^{* *}$ & 3,2 & $10,9^{* *}$ & & $0,8^{* *}$ \\
\hline 1,0 & 2,50 & . & - & - & - & - & - & - & - & - & - & $0,2^{* *}$ & - \\
\hline 1,7 & 4,25 & - & - & - & - & - & - & $0,2^{* *}$ & - & - & - & - & - \\
\hline 2,5 & 6,37 & $10,9^{*}$ & $10,9^{*}$ & $6,5^{*}$ & $10,2^{*}$ & $1,4^{* *}$ & $67,0^{*}$ & - & $66,5^{*}$ & $0,6^{* *}$ & $25,7^{*}$ & $5,5^{*}$ & $18,1^{*}$ \\
\hline
\end{tabular}

$V_{1}$ - velocidad; $Q_{1}$ - caudal principal; DP - presión diferencial entre la entrada y la garganta $\left(P_{1}-P_{2}\right) ; \Delta h_{v}$ - pérdidas totales en el inyector $\left(P_{1}-P_{3}\right) ;\left(P_{3}-P_{2}\right)$ - presión diferencial entre la garganta y la salida; "error máximo; "*error mínimo

mayor es la velocidad y la distorsión del flujo aumenta con la cavitación.

La presión mínima en el eje del Venturi no se produce exactamente en el punto de unión del eje de la aspiración $(X=0)$, sino que se encuentra ligeramente desplazada en la dirección aguas abajo.
En el perímetro de unión tobera-garganta y gargantadifusor, ambos modelos muestran una zona de disminución de la presión, probablemente debida a las uniones en arista viva. A partir de un cierto grado de desarrollo de la cavitación, es decir por debajo de una determinada presión en la garganta, 

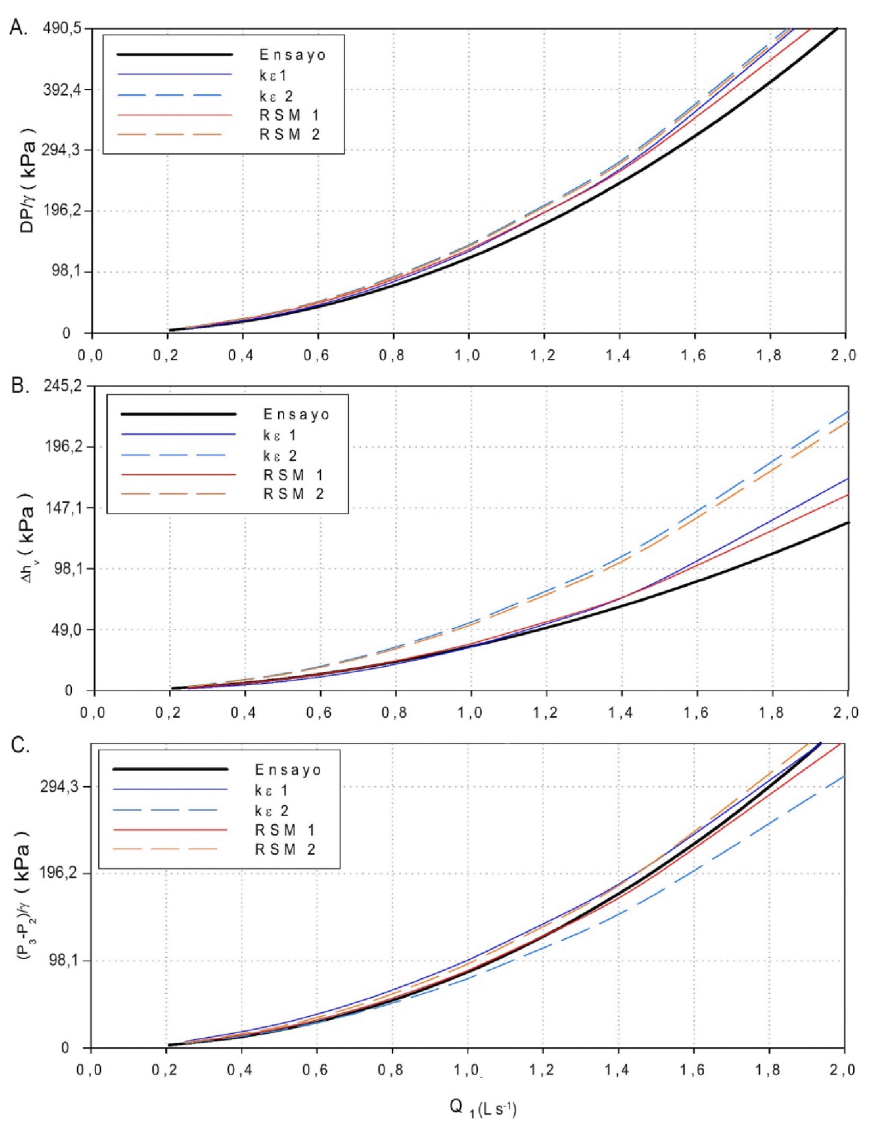

Figura 11. Presión diferencial entre la entrada y la garganta (DP) (A), pérdidas totales en el inyector $\left(\Delta \mathrm{h}_{\mathrm{v}}\right)$ (B) y presión diferencial entre la garganta y la salida $\left(\mathrm{P}_{3}-\mathrm{P}_{2}\right)(\mathrm{C})$ frente al caudal principal $\left(\mathrm{Q}_{1}\right)$ y comparativa de los modelos CFD con el ensayo, para el prototipo v4-50-0,2-6

los modelos utilizados no son capaces de proporcionar valores coherentes de presión y velocidad.

Así como en este ensayo, Gibson et al. (1999) y ReaderHarris et al. (2001) utilizaron modelos K- $\varepsilon$ y RSM en el análisis de caudalímetros Venturi, consiguiendo buenos ajustes a los datos experimentales con ambos, pero principalmente con el último. Los trabajos versaban sobre la influencia de los orificios de toma de presión en caudalímetros, puesto que Frankling \& Wallance (1970) habían comprobado que la presión detectada por sensores se ve ligeramente influenciada, al alza, por los orificios.

En los trabajos que analizaron el fenómeno de la cavitación en Venturis, como de Delgosha et al. (2003b) y de Vortmann et al. (2003), los modelos de turbulencia empleados con buenos resultados fueron k- $\varepsilon, \mathrm{k}-\omega$ y RSM. Palau-Salvador et al. (2007) validaron modelo de cavitación de FLUENT, modelando varios orificios, estrechamientos y Venturis, comparandolo con los resultados experimentales. Los autores concluyeron que el modelo de cavitación empleado simula adecuadamente la cavitación del flujo a través de geometrías sencillas, como los orificios y estrechamientos, en estado estacionario.

En estos experimentos comparados con modelos CFD se constató el comportamiento inestable de la cavitación y su alta dependencia del modelo de turbulencia seleccionado. Al igual que en el presente trabajo también fue comprobada la dificultad de reproducir computacionalmente los valores experimentales en presencia de cavitación.

\section{Fase E2 - inyección inferior}

En la Figura 12 se muestra la representación gráfica de los caudales de aspiración frente a los principales, para los modelos RSM y k- $\varepsilon$, en cada prototipo.

El caudal máximo inyectado es tanto menor cuanto mayor es la presión de entrada. La explicación es que la presión en la garganta es tanto menor cuanto mayor es $\left(Q_{1}+q\right)$ y con mayores presiones $P_{1}$ los caudales de entrada son mayores. Para un mismo caudal $\left(Q_{1}+q\right)$, correspondiente a otras presiones de entrada distintas, el caudal inyectado es menor cuando se alcanza la cavitación con la presión más alta.

Dentro de los límites de presión establecidos, los resultados de caudal inyectado obtenidos en las simulaciones alcanzan valores inferiores que los experimentales, en general el modelo RSM predice mejor la inyección que el modelo K- $\varepsilon$.

Tabla 6. Error relativo de los modelos CFD, en el prototipo V3-50-0,38-6

\begin{tabular}{|c|c|c|c|c|c|c|c|c|c|c|c|c|c|}
\hline \multirow{2}{*}{$\begin{array}{c}V_{1} \\
\left(m^{-1}\right)\end{array}$} & \multirow{2}{*}{$\begin{array}{c}a_{1} \\
\left(L s^{-1}\right)\end{array}$} & \multicolumn{4}{|c|}{ DP (\%) } & \multicolumn{4}{|c|}{$\Delta h_{v}(\%)$} & \multicolumn{4}{|c|}{$\mathbf{P}_{3}-\mathbf{P}_{2}(\%)$} \\
\hline & & $k-\varepsilon 1$ & $k-\varepsilon 2$ & RSM 1 & RSM 2 & $k-\varepsilon 1$ & $k-\varepsilon 2$ & RSM 1 & RSM 2 & $k-\varepsilon 1$ & $k-\varepsilon 2$ & RSM 1 & RSM 2 \\
\hline $\begin{array}{l}0,5 \\
0,9\end{array}$ & $\begin{array}{l}0,80 \\
15\end{array}$ & $11,3^{*}$ & $19,6^{\star}$ & $10,9^{*}$ & $19,4^{*}$ & $12,4^{*}$ & $4,7^{* *}$ & $7,0^{*}$ & $4,9^{\star \star}$ & $\overline{-}^{-}$ & $1,4^{\star \star}$ & & $1,6^{* *}$ \\
\hline $\begin{array}{l}0,9 \\
3,5\end{array}$ & $\begin{array}{l}1,0 \\
5,6\end{array}$ & $4,2^{* *}$ & $11,9^{* *}$ & $4,4^{* *}$ & $12,0^{* *}$ & - & $28,4^{*}$ & $6,4^{* *}$ & $23,7^{*}$ & $\begin{array}{l}0,5^{* x} \\
20,9^{*}\end{array}$ & - & $\begin{array}{l}0,3^{* *} \\
20,5^{*}\end{array}$ & $28,6^{*}$ \\
\hline
\end{tabular}

$V_{1}$ - velocidad; $Q_{1}$ - caudal principal; DP - presión diferencial entre la entrada y la garganta $\left(P_{1}-P_{2}\right) ; \Delta h_{v}$ - pérdidas totales en el inyector $\left(P_{1}-P_{3}\right) ;\left(P_{3}-P_{2}\right)$ - presión diferencial entre la garganta y la salida; "error máximo; "*error mínimo

Tabla 7. Error relativo de los modelos CFD, en el prototipo V4-50-0,2-6

\begin{tabular}{|c|c|c|c|c|c|c|c|c|c|c|c|c|c|}
\hline \multirow{2}{*}{$\begin{array}{c}V_{1} \\
\left(m s^{-1}\right)\end{array}$} & \multirow{2}{*}{$\begin{array}{c}a_{1} \\
\left(L s^{-1}\right)\end{array}$} & \multicolumn{4}{|c|}{ DP (\%) } & \multicolumn{4}{|c|}{$\Delta h_{v}(\%)$} & \multicolumn{4}{|c|}{$P_{3}-P_{2}(\%)$} \\
\hline & & $k-\varepsilon 1$ & $k-\varepsilon 2$ & RSM 1 & RSM 2 & $k-\varepsilon 1$ & $k-\varepsilon 2$ & RSM 1 & RSM 2 & $k-\varepsilon 1$ & k- 22 & RSM 1 & RSM 2 \\
\hline 0,5 & 0,8 & $18,1^{*}$ & $24,1^{*}$ & $15,5^{\star}$ & $22,4^{*}$ & & $51,2^{* *}$ & $3,2^{* *}$ & $45,6^{\star \star}$ & $24,8^{*}$ & $31,1^{*}$ & $15,6^{\star}$ & $25,4^{*}$ \\
\hline 0,6 & 1,0 & - & - & - & - & $1,1^{* *}$ & - & - & - & - & - & - & - \\
\hline 0,8 & 1,3 & - & - & - & - & - & - & - & - & - & - & $0,9^{* *}$ & - \\
\hline 0,9 & 1,5 & - & - & - & - & - & - & - & - & $0,6^{* *}$ & - & - & $2,4^{* *}$ \\
\hline 1,2 & 2,0 & $0,7^{* *}$ & $7,4^{* *}$ & $0,4^{* *}$ & $6,2^{* *}$ & $26,1^{*}$ & $66,2^{*}$ & $16,5^{*}$ & $60,0^{*}$ & - & $2,2^{\star *}$ & - & 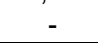 \\
\hline
\end{tabular}

$V_{1}$ - velocidad; $Q_{1}$ - caudal principal; DP - presión diferencial entre la entrada y la garganta $\left(P_{1}-P_{2}\right) ; \Delta h_{v}$ - pérdidas totales en el inyector $\left(P_{1}-P_{3}\right) ;\left(P_{3}-P_{2}\right)$ - presión diferencial entre la garganta y la salida; "error máximo; "*error mínimo 

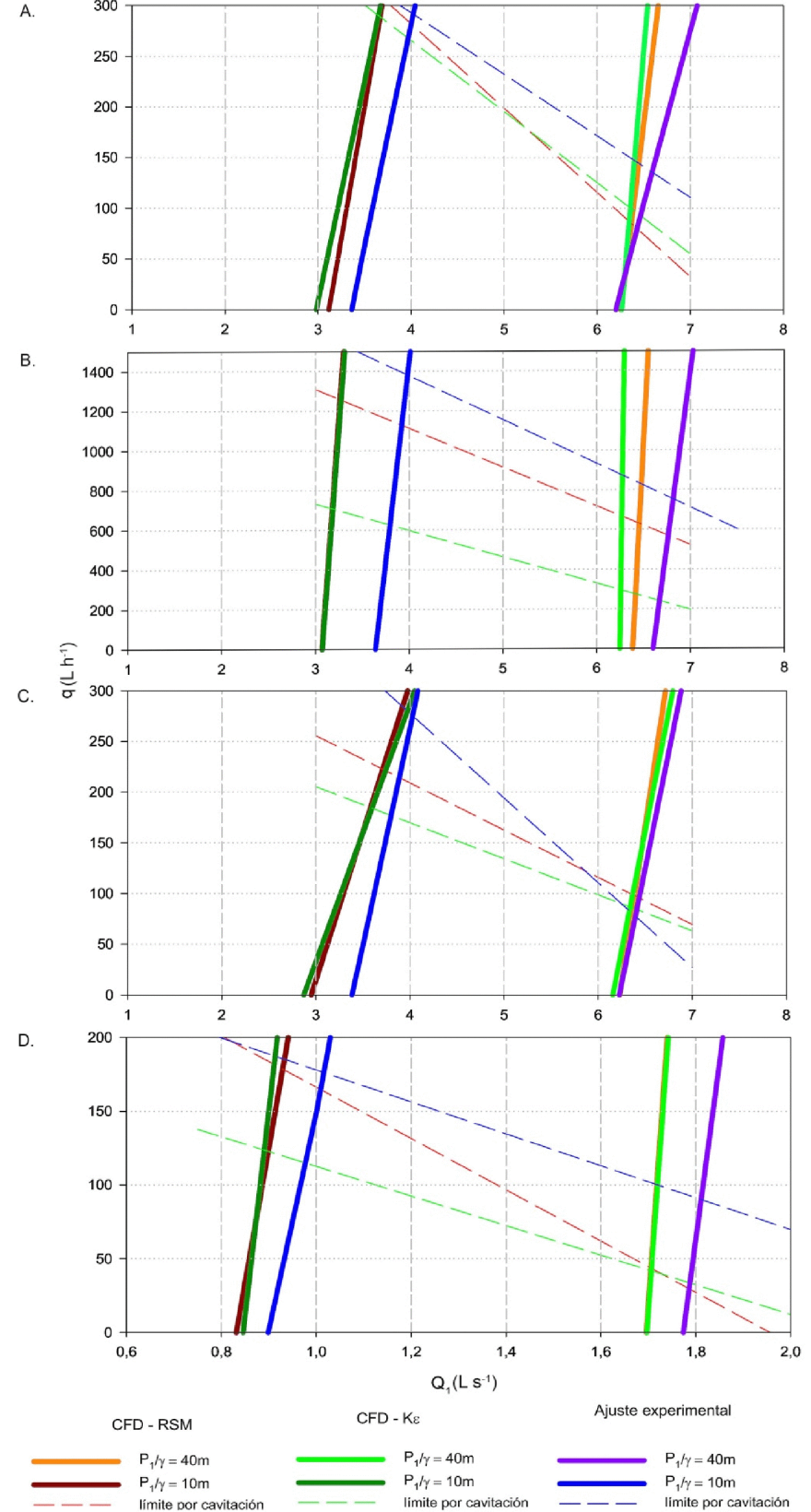

Figura 12. Caudal inyectado (q) frente al caudal principal $\left(\mathrm{Q}_{1}\right)$, para los métodos CFD y datos experimentales, en el prototipo V1-63-0,3-6 (A), V263-0,3-16 (B), V3-63-0,38-6 (C) y V4-63-0,2-6 (D)

Al igual que en este ensayo, la idoneidad de las técnicas CFD para describir el comportamiento hidráulico de inyectores Venturi ha sido comprobada por Manzano \& Palau (2005), una vez que los resultados experimentales y los obtenidos por ordenador fueron similares.

Sun \& Niu (2012) utilizaron las técnicas CFD para estudiar los efectos de parámetros geométricos (relación entre longitud y diámetro de la garganta $(\lambda)$ y entre diámetros de la garganta y tobera $(\beta)$ en el funcionamiento de un inyector Venturi (velocidad media de salida, presiones mínima y critica, coeficiente de pérdida de carga localizada y relación de inyección de fertilizante), aunque sin llegar a validar experimentalmente las simulaciones realizadas. Los resultados indicaron que la presión mínima se produjo en la pared de entrada de la garganta y existiendo una relación lineal entre la presión mínima y la diferencia de presión en la entrada y la salida. La relación de contracción de la garganta fue el principal factor en el efecto del rendimiento de inyector Venturi. En un análisis sobre los mismos parámetros geométricos de un Venturi de $30 \mathrm{~mm}$ de diámetro de entrada y usando también modelos de turbulencia $\mathrm{K}-\varepsilon$, Huang et al. (2009) verificaron sobre un único punto de funcionamiento experimental un error del 5,6\% entre el caudal real inyectado y el caudal obtenido por cálculo numérico.

Baylar et al. (2009) estudiaron los efectos de algunos parámetros geométricos en las tasas de inyección de aire por con Venturi mediante técnicas CFD, empleando el programa FLUENT. Los autores concluyeron que existía suficiente proximidad entre las tasas de inyección de aire medidas y los valores calculados a partir del programa, con errores medios entre 6 y $13 \%$.

\section{Conclusiones}

1. Las técnicas CFD exigen un buen ajuste del modelo para dar un resultado aceptable.

2. Son interesantes para comparar geometrías, analizar sus variantes, realizar prediseños y aproximar ordenes de magnitud, pero es imprescindible el ensayo, especialmente en las proximidades de la cavitación.

\section{Literatura Citada}

Baylar, A.; Aydin, M. C.; Unsal, M.; Ozkan, F. Numerical modeling of Venturi flows for determining air injection rates using Fluent v.6.2. Mathematical and Computational Applications, v.14, p.97-108, 2009.

Cipolla, E. Z.; Silva, F. das G. B. da.; Tiago Filho, G. L.; Barros, R. M. Avaliação da distribuição de velocidades em uma bomba centrífuga radial utilizando técnicas de CFD. Revista Brasileira de Recursos Hídricos, v.16, p.71-79, 2011.

Davis, J. A.; Stewart, M. Predicting globe control valve performance. Part I: CFD modelling. Journal of Fluids Engineering, v.124, p.772-777, 2002.

Delgosha, O. C.; Fortes, P.; Reboud, J. L. Evaluation of the turbulence model influence on the numerical simulations of unsteady cavitation. Journal of Fluids Engineering, v.125, p.38-45, 2003a.

Delgosha, O. C.; Reboud, J. L.; Delannoy, Y. Numerical simulation of the unsteady behaviour of cavitating flows. International Journal for Numerical Methods in Fluids, v.42, p.527-548, 2003 b.

Frankling, R. E.; Wallace, J. M. Absolute measurements of statichole error using flush mounted transducer. Journal of Fluid Mechanics, v.42, p.33-48,1970.

Gibson, J. J.; Reader-Harris, M. J.; Gilchrist, A. CFD analysis of the static-hole error caused by tappings in Venturimeters operating in high-pressure gas. In: ASME/JSME, 3, 1999, Joint Fluids Engineering Conference, San Francisco FEDSM 99-7149. Proceedings... NewYork: American Society of Mechanical Engineers.1999. s.p. 
Guo, B.; Lengrish, T. A .G.; Fletcher, D. F. CFD Simulation of precession in Sudden sudden pipe expansion with low inlet swirl. Applied Mathematical Modelling, v.26, p.1-15, 2002.

Hatano, S.; Kang, D.; Kagawa, S.; Nohmi, M.; Yokota, K. Study of cavitation instabilities in double-suction centrifugal pump. International Journal of Fluid Machinery and Systems, v.7, p.94-100, 2014.

Huang, K.; Li, G.; Wang, H. CFD simulation to the flow field of Venturi Injector. In: Li, D.; Chunjiang, Z (ed.). Computer and computing technologies in agriculture II. Boston: Springer, v.2, 2009. p.805-815.

Lindau, J. W.; Kunz, R. F.; Boger, D. A.; Stinebring, D. R.; Gibeling, H. J. High Reynolds number, unsteady, multiphase CFD modeling of cavitating flows. Journal of Fluids Engineering, v.124, p.607-616, 2002.

Manzano, J. Análisis del inyector Venturi y mejora de su instalación en los sistemas de riego localizado. Valencia: UPV, 2008. 248p. Tesis Doctoral

Manzano, J.; Palau, G. Hydraulic modelling of Venturi injector by means of CFD. In: ASABE Annual International Meeting, 2005, Tampa. Proceedings... Tampa: ASABE, Paper number 052070. 2005. s.p.

Norton, T.; Sun, D. W.; Grant, J.; Fallon, R.; Dodd, V. Applications of computational fluid dynamics (CFD) in the modeling and design of ventilation systems in the agricultural industry: A review. Bioresource Technology, v.98, p.2386-2414, 2007.

Palau-Salvador, G.; González-Altozano, P.; Arviza-Valverde, J. Numerical modeling of cavitating flows for simple geometries using FLUENT v.6.1. Spanish Journal of Agricultural Research, v.5, p.460-469, 2007.
Palau-Salvador, G.; González-Altozano, P.; Arviza-Valverde, J. Three-dimensional modeling and geometrical influence on the hydraulic performance of a control valve. Journal of Fluids Engineering, v.130, p.1-9, 2008.

Reader-Harris, M. J.; Brunto, W. C.; Gibson, J. J.; Hodges, D.; Nicholson, I. G. Discharge coefficients of Venturi tubes with standard and non-standard convergent angles. Flow Measurement and Instrumentation, v.12, p.135-145, 2001.

Shingal, A. K.; Athavale, M. M.; Li, H.; Jiang, Y. Mathematical basis and validation of the full cavitation model. Journal of Fluids Engineering, v.124, p.617-624, 2002.

Sun, Y.; Niu, W. Simulating the effects of structural parameters on the hydraulic performances of Venturi tube. Modelling and Simulation in Engineering, v.1, p.1-7, 2012.

Teruel, B. J. Controle automatizado de casas de vegetação: Variáveis climáticas e fertigação. Revista Brasileira de Engenharia Agrícola e Ambiental, v.14, p.237-245, 2010.

Vortmann, C.; Schnerr, G. H.; Seelecke, S. Thermodynamic modelling and simulation of cavitating nozzle flow. International Journal of Heat and Fluid Flow, v.24, p.774783, 2003.

Wei, Q.; Shi, W.; Dong, W.; Lu, G.; Huang, S. Study on hydraulic performance of drip emitters by computational fluid dynamics. Agricultural Water Management, v.84, p.130136, 2006.

Wendt, J. F. Computational fluid dynamics: An introduction. Berlin: Springer-Verlag. 2009. 333p.

Xing, T.; Frankel, S. H. Effect of cavitation on vortex dynamics in a submerged laminar jet. AIAA Journal, v.40, p.22662276, 2002.

Yeoh, G. H.; Liu, C.; Tu, J.; Timchenko, V. Computational Fluid Dynamics and its applications. Modelling and Simulation in Engineering, v.1, p.1-2, 2012. 